\title{
Redesenho gerencial do processo educativo da equipe de enfermagem em hospital pediátrico de nível terciário
}

Managerial redesign of the educational process of the nursing team the tertiary-level pediatric hospita

Rediseño gerencial del proceso educativo del equipo de enfermería en hospital pediátrico

Amparito del Rocío Vintimilla Castro ${ }^{1}$ (D) https://orcid.org/000-0002-7581-6915

Ana Paula Almeida ${ }^{1}$ (1) https://orcid.org/000-0001-5234-4508

Ana Paula Fraga ${ }^{1}$ (1) https:// orcid.org/0000-0002-1853-8250

\section{Resumo}

0 processo sistematizado para treinamento dos profissionais ingressantes auxilia nas necessidades educativas de saúde relacionadas à segurança do paciente. Este estudo propõe-se a descrever a experiência de redesenho de programa educativo dirigido a profissionais de enfermagem ingressantes em hospital-escola pediátrico de nível terciário da cidade de São Paulo e a apresentar considerações sobre os resultados dessa experiência, no que se refere às notas do pré e do pós-teste, para verificar se o novo desenho de treinamento favoreceu 0 aprendizado dos conteúdos propostos. Trata-se de relato de experiência fundamentado à luz dos conceitos de gerenciamento do conhecimento de Nonaka. Os profissionais apontaram satisfação com o acolhimento diferenciado e relataram sentimento de segurança para iniciar as atividades nas unidades para prestarem assistência de qualidade em pediatria. Houve diferenças relevantes das médias das notas entre os pré e pós-testes. 0 redesenho do programa de capacitação evidenciou eficácia e maior envolvimento dos profissionais ingressantes.

\section{Abstract}

A systematic process for training new professionals that helps with educational health needs related to patient safety. This study aims to describe the experience of redesigning an educational program for nursing professionals entering a pediatric teaching hospital at a tertiary level in the city of São Paulo. As well as to present considerations on the results of the experience regarding pre and post-test to verify if the new training favors the learning proposals. This research is an experience report based on Nonaka's knowledge management concepts. The professionals pointed out satisfaction with the differentiated reception and reported a safety feeling to start the activities in the units to provide quality care in pediatrics. There were significant differences in the average scores between the pre and post-tests. The redesign of the training program indicated the effectiveness and greater involvement of the new professionals.

\section{Resumen}

Um proceso sistematizado para entrenamiento de los profesionales ingresantes auxilia em las necesidades educativas de salud relacionadas a la seguridad del paciente. Este estúdio tiene como objetivo describir un rediseñado del proceso educativo a los profesionales de enfermería recién contratados en hospital pediátrico de nível terciário de la ciudad de São Paulo-Brasil y verificar si el modelo propuesto fue eficaz. Este es un relato de experiencia fundamentado a la luz de los conceptos de gerenciamento del conocimiento de Nonaka. Los profesionales indicaron satisfacción con la recepción diferenciada e informaron una sensación de seguridad al iniciar actividades en las unidades para proporcionar atención de calidad en pediatría. Encontramos diferencias relevantes entre el promedio de las pruebas previas y posteriores. El rediseño del programa de capacitación evidenció eficacia y mayor envolvimiento de los profesionales ingresantes.

\section{Como citar:}

Castro AR, Almeida AP, Fraga AP. [Managerial redesign of the educational process of the nursing team the tertiary-level pediatric hospital]. Rev Soc Bras Enferm Ped. 2020;20(1):54-9. Portuguese

\footnotetext{
${ }^{1}$ Instituto da Criança, Hospital das Clínicas, Faculdade de Medicina, Universidade de São Paulo, São Paulo, SP, Brasil.
}

Conflitos de interesse: nada a declarar.

Submetido: 17 de Janeiro de 2019 | Aceito: 30 de Junho de 2020

Autor correspondente: Amparito del Rocío Vintimilla Castro | E-mail: amparito.castro@hc.fm.usp.br

Dol: http://dx.doi.org/10.31508/1676-3793202000008

\section{Descritores \\ Enfermagem pediátrica; Tutorial; Educação permanente}

Keywords

Pediatric nursing; Mentoring; Education continuing

\section{Descriptores}

Enfermería pediátrica; Tutoría; Educación continua 


\section{Introdução}

Adotar práticas de acolhimento e valorização do profissional ingressante contribui para o atendimento das necessidades educativas e organizacionais sem esquecer o aspecto humano. As constantes mudanças no mercado de trabalho, o avanço tecnológico e a necessidade de profissionais cada vez mais qualificados e habilitados tornam-se requisitos para assegurar maior e melhor qualidade nos resultados da assistência aos clientes, para garantir a segurança do paciente e do próprio profissional. Essas convergências de propósitos educativos vão ao encontro da missão, da visão e dos valores de uma instituição de saúde.

O grande volume de conhecimentos gerados na área de saúde e o contínuo movimento de inovação tecnológica vêm exigindo dos dirigentes dessas organizações uma busca constante de competência e superação dos atuais padrões assistenciais. ${ }^{(1)} \mathrm{A}$ questão do treinamento, do aprimoramento e da formação dos profissionais da área de saúde inscreve-se no contexto de avanços e propostas educativas de forma individualizada, com impacto no diferencial competitivo das instituições, enriquecendo o quadro de valores dos profissionais e a empregabilidade. O conhecimento subsidia as habilidades e as competências do saber, fazer e saber ser. Esses elementos acompanham o profissional e exigem dele capacitação técnica, recurso-chave das empresas, bem como investimento pessoal, que fortalece a educação e os programas de treinamento. ${ }^{(2)}$ Para tal, acredita-se na necessidade de proporcionar o empowerment education, ou empoderamento educativo, com base na busca de conhecimento do próprio profissional, para contribuir com sua "emancipação" e seu desenvolvimento de habilidades e com o conhecimento de suas atividades profissionais de forma crítico-reflexiva. ${ }^{(3,4)}$

É comum que o profissional ingressante tenha medos, expectativas e insegurança em relação a um ambiente desconhecido. Por isso, faz-se essencial a prática da humanização do facilitador, relacionada à flexibilidade, à criatividade, ao compromisso, ao dinamismo, ao bom relacionamento interpessoal e à disposição para atualização permanente.

A capacitação é a preparação do profissional com ações que integrem o ensino à prática profissional, para o desempenho de suas atividades, de forma crítica e refle- xiva, capaz de promover tanto o desenvolvimento cognitivo quanto as habilidades técnicas e atitudes éticas. Essa capacitação contempla o auxílio para a integração, a adaptação e o compromisso com os resultados do trabalho. ${ }^{(5)}$ Para contemplar uma capacitação que atenda às necessidades dos profissionais ingressantes e à própria instituição, é fundamental que os serviços de educação permanente redesenhem os programas de capacitação, ou seja, busquem incorporar melhorias de um programa existente, e que sejam capazes de provocar melhorias significativas, para atingir objetivos e metas das organizações, em termos da segurança do paciente.

A filosofia e as ações do Serviço de Educação Permanente (SEP), pautadas na constante busca de conhecimento e ideias inovadoras adequadas às exigências atuais e futuras do mercado de trabalho, contribuem para que o profissional ingressante tenha condições de atuar de acordo com os propósitos da instituição, nivelando-o em conhecimento teórico-prático. Desta forma, veem-se alternativas de transpor o modo tradicional de educação com atividades inseridas no contexto sociocultural, econômico, político e ético. ${ }^{(6)}$

Assim, considerando a responsabilidade social de melhorar a capacitação de profissionais de enfermagem e estimular o desenvolvimento pessoal e a autoestima, é fundamental a implantação de um programa de capacitação para a segurança do paciente pediátrico e do próprio profissional que atenda também a Política Nacional de Educação Permanente em Saúde.(7)

Um programa de educação permanente deve ser disseminado em relação à sua concepção, abranger ações de modo a atender às necessidades da clientela, dos profissionais, dos gestores e da instituição ${ }^{(8)} \mathrm{e}$ ser importante para o gestor, no sentido de impulsionar ao constante desenvolvimento e à capacitação dos profissionais para a atenção em saúde. ${ }^{(9)}$

Com intuito de melhorar a capacitação de profissionais de enfermagem, com estímulo ao desenvolvimento pessoal e à autoestima dos colaboradores ingressantes, este estudo objetivou descrever a experiência de redesenho de um programa educativo aos profissionais de enfermagem ingressantes em um hospital-escola pediátrico de nível terciário e apresentar considerações sobre os resultados da experiência, no que se refere às notas do pré e do pós-teste, para verificar se o novo treinamento favoreceu o aprendizado dos conteúdos propostos. 


\section{Métodos}

Trata-se de um relato de experiência do redesenho do programa educativo destinado a profissionais de enfermagem ingressantes fundamentado à luz dos conceitos de gerenciamento do conhecimento de Nonaka, ${ }^{(10)}$ que é uma das mais conhecidas teorias da construção do conhecimento.

Baseia-se em quatro fases, conhecidas como socialização, externalização, combinação e internalização (SECI), as quais possibilitam a mudança dos conhecimentos tácito e explícito. ${ }^{(10,11)}$ A socialização corresponde ao momento em que ocorrem a troca e o compartilhamento de conhecimentos entre os integrantes de um grupo, por meio do conhecimento tácito, subjetivo ou da experiência individual. Cria-se, então, um ambiente de discussão, reflexão e esclarecimento de dúvidas. A externalização se dá quando novos conhecimentos, os explícitos, são aprendidos, com base no conhecimento tácito dos integrantes de um grupo. A combinação refere-se ao conhecimento prévio e aos conhecimentos recém-aprendidos, resultando na criação de novos conhecimentos; trata-se da conversão de conhecimento explícito em novos conhecimentos explícitos. Já a internalização é o momento em que acontece a difusão interativa do conhecimento explícito em tácito pela repetição de tarefas em um momento de aprendizagem e socialização. Inicia-se aí um novo ciclo de criação de conhecimentos.

Tanto a externalização quanto a internalização são os modos de conhecimento explícito e objetivo desenvolvidos por meio da racionalidade e complementados mutuamente. Entendem-se esses conhecimentos como aqueles que podem ser utilizados em favor da instituição e inseridos em suas atribuições profissionais. Esses conceitos de gerenciamento do conhecimento foram aplicados para redesenhar o programa educativo do SEP de um hospital de grande porte da cidade de São Paulo.

\section{Contextualização e operacionalização da experiência}

Antes da aplicação do redesenho desse programa, o treinamento era realizado de forma tradicional e contemplava aulas expositivas e demonstração de técnicas de enfermagem, com avaliação de pré e pós-teste com maior participação do enfermeiro do SEP e participação passiva do colaborador. Para a aplicação desse programa redesenhado, foram utilizadas metodologias de ensino que favoreceram e apoiaram o desenvolvimento do programa, listando o gerenciamento do conhecimento pautado por Nonaka: ${ }^{(10)}$ dramatizações, dinâmicas de grupo, aulas dialogadas com leituras prévias e aulas expositivas. ${ }^{(12)}$

As fases de gerenciamento do conhecimento de Nonaka ${ }^{(10)}$ aplicadas no redesenho do programa de treinamento admissional de um hospital-escola de nível terciário são apresentadas a seguir:

- Socialização: aplicado desde o primeiro dia em que o profissional é encaminhado ao SEP. Nesse dia, iniciou-se o acolhimento, criando-se uma roda para as apresentações dos colaboradores recém-admitidos e para a equipe do SEP estimular a descontração dos participantes. Apresentou-se um vídeo institucional e foi dada orientação sobre aspectos pertinentes a horários, assiduidade, pontualidade e apresentação pessoal, além de esclarecimento de dúvidas. Realizou-se um tour pelos diferentes setores da instituição. Explicaram-se e analisaram-se os direitos, os deveres e as atribuições de cada categoria profissional, por meio de material impresso.

- Combinação: a sondagem de conhecimentos prévios e as percepções dos colaboradores recém-admitidos foram documentadas em pré-teste com assuntos relacionados a prevenção de infecção (cuidado limpo é cuidado seguro com a higienização das mãos); cálculo e diluições de medicamentos; comunicação eficaz (anotação de enfermagem); infusão de sangue e hemocomponentes; manipulação de cateteres, conexões e conectores; cateterismo enteral; prevenção de quedas; passos para a segurança na administração de medicamentos e perguntas sobre segurança do paciente.

- Externalização: os novos conhecimentos para a prática segura do paciente pediátrico foram realizados a partir dos assuntos mencionados. Além destes, incluíram-se o Estatuto da Criança e do Adolescente; ética aplicada à enfermagem; crescimento e desenvolvimento humano; importância da família na recuperação da criança hospitalizada; humanização; morte na infância; sinais vitais em pediatria; doenças respiratórias e gastrintes- 
tinais; identificação da graduação de seringas de diferentes volumes; manipulação e programação de bomba de infusão para medicação e dieta enteral; identificação e cuidados com aparelhos para aferição da pressão arterial manual e eletrônica; planejamento da assistência de enfermagem e comunicação e relacionamento interpessoal. Estes assuntos foram trabalhados por meio de estudos de caso, práticas em laboratório, trabalhos em grupos, simulações, aprendizagem baseada em problemas e dinâmicas de grupo. A comunicação, a reflexão e as discussões estão presentes em todos os momentos do processo educativo.

- Internalização: a difusão interativa dos assuntos propostos aconteceu com auxílio de metodologias ativas, nas quais os profissionais eram os autores principais, tornando o processo de aprendizado mais dinâmico, pela autonomia e pela mudança de atuação do enfermeiro do SEP. Este era o mediador e o estimulador de conhecimentos, embasado no diálogo problematizador, com contextualização e aplicação prática. Para tal, abriu-se um espaço de diálogo para aprendizado de conteúdos fornecidos para leitura, discussão e troca de informações reforçadas ou esclarecidas pelo tutor. As fases de gerenciamento do conhecimento de Nonaka ${ }^{(10)}$ aplicadas foram sistematizadas para o treinamento de enfermeiros e técnicos de enfermagem ingressantes. Os assuntos abordados relacionaram-se ao cuidado do neonato, da criança e do adolescente acolhidos nas unidades de internação e ambulatório, bem como em unidades de apoio (centro de material e esterilização, centro cirúrgico, serviço de diagnóstico e apoio terapêutico). Os assuntos foram selecionados e extraídos tanto da experiência dos enfermeiros da educação permanente quanto dos gestores das unidades assistenciais e dos propósitos da instituição. Os profissionais recém-contratados foram acolhidos e submetidos a esse programa, que abrangeu avaliação diária, para trabalhar as dificuldades apresentadas devido aos diferentes níveis de conhecimento desses profissionais. Manteve-se a devolutiva constante, com reforços e estímulo contínuos, preparando-os para a assistência ao cliente pediátrico de alta complexidade. Esse programa foi executado em 10 dias, com total de 60 horas.

Foi mantida, conforme desenho do treinamento já existente, a avalição dos conhecimentos com pré e pós-testes sobre os assuntos abordados, sinalizando os pontos fortes e os de melhoria para estímulo dos colaboradores. Ao finalizar o treinamento, solicitou-se uma avaliação sobre o acolhimento e o treinamento oferecidos pelo SEP. Todas as informações do processo educativo do colaborador ingressante foram registradas em impressos próprios, como parte do prontuário do profissional. Esse prontuário foi encaminhado ao gerente da unidade, e o colaborador foi designado para a continuação da avaliação durante o período probatório de 3 meses.

Dessa forma, a aplicação das quatro fases da teoria do conhecimento de Nonaka ${ }^{10}$ foi complementada com as quatro fases de procedimentos já realizados pelo SEP antes desse redesenho: avalição do conhecimento por meio de pré e pós-testes, narrativas e avaliação do treinamento por parte dos colaboradores, finalizando com o registro de todo esse processo nos prontuários dos novos colaboradores.

\section{Resultados}

Para este relato, considerou-se a experiência de janeiro de 2010 a dezembro de 2017. Elaborou-se um cronograma de atividades por dia, para atender os objetivos propostos, cumprindo os prazos e a carga horária preestabelecidos. No período, 237 profissionais participaram do programa, sendo 131 enfermeiros e 106 técnicos de enfermagem.

Após o programa educativo da equipe de enfermagem, chamaram-nos a atenção as notas de alguns conteúdos, com diferenças relevantes antes e depois do redesenho gerencial de treinamento. Em relação às notas dos enfermeiros, ressaltam-se: anotações de enfermagem (comunicação efetiva), que atingiram 4,7 no pré e 7,9 no pós-teste; prevenção de infecção (cuidado limpo é cuidado seguro), com 6,5 no pré-teste e 9,6 no pós-teste; cálculo de medicamentos, com 3,4 no pré-teste e 8,9 no pós-teste e segurança do paciente, com 6,5 no pré e 9,5 no pós-teste. No que diz respeito aos técnicos de enfermagem, as notas dos pré e pós-testes foram, respectivamente, em anotações de enfermagem, 4,4 e 8,0; em prevenção de infeção, 5,7 e 9,7; em cálculo de medicamentos, 3,7 e 8,2 e em segurança do paciente, 3 e 8,1.

Quanto à avaliação dos colaboradores sobre o treinamento recebido, foi apontada satisfação com o 
acolhimento e o treinamento diferenciado e organizado, bem como sentimento de segurança para iniciar as atividades nas unidades, com a finalidade de elas prestarem uma assistência de qualidade em pediatria, além da importância de serem estimulados a trabalharem em equipe e manterem comunicação eficaz. Importante pontuar que foi observado o envolvimento dos profissionais ingressantes com o processo admissional sistematizado baseado na construção do conhecimento.

\section{Discussão}

O conhecimento só é criado pelas pessoas ou por seus colaboradores e, dessa forma, uma instituição tem condições de crescimento de produção intelectual com a participação de seus colaboradores. Partindo desse princípio, a instituição precisa apoiar e valorizar os profissionais, propiciando condições para o desenvolvimento e a atenção das expectativas e demandas de seus clientes. ${ }^{(10)} \mathrm{A}$ aplicação de um redesenho gerencial que sustente o processo educativo dos colaboradores recém-admitidos, utilizando um referencial teórico, como a teoria de Nonaka, ${ }^{(10)}$ que mantém fases sistematizadas, permite atender às necessidades educativas dos novos profissionais que assistirão o cliente neonatal e pediátrico. $\mathrm{O}$ aprendizado dos profissionais ingressantes é notório pelos bons resultados dos pós-testes, o que sugere o desenvolvimento de habilidades cognitivas no treinamento admissional e a repercussão de sua satisfação durante o período que permanecem em um SEP.

A prática do processo de gerenciamento do conhecimento é eficaz para o alinhamento técnico dos colaboradores e contribui para o aprendizado dentro da instituição e a satisfação dos clientes atendidos. ${ }^{(13)}$ Ao propiciar a externalização da experiência do colaborador, inserido no grupo de treinamento, a criação de novos conhecimentos compartilhados com o grupo foi enriquecida e facilitada.

A aplicação de bases teóricas direcionadas às necessidades da clientela pediátrica permite dar oportunidade de internalização da experiência individual, das crenças, dos valores e do conhecimento compartilhado, de modo formal, aliado também aos documentos e aos protocolos institucionais, para posterior sedimentação do conhecimento no aprender fazendo. ${ }^{(13,14)}$
$\mathrm{O}$ registro das atividades de treinamento e o encaminhamento até a unidade de trabalho são necessários para o acompanhamento do colaborador em suas atividades laborais, com a identificação de seus pontos fortes e os de melhoria, o que pode contribuir com uma avaliação mais crítica e assertiva do gerente, que irá acompanhá-lo até o final do período probatório. ${ }^{(13)}$

$\mathrm{O}$ ambiente físico e os recursos materiais fazem parte do treinamento, no qual os colaboradores possam ser acolhidos em condições adequadas para o desenvolvimento do processo educativo, pautado no redesenho gerencial proposto. ${ }^{(15)}$ São necessárias a alocação e a organização de uma área física com laboratório de simulação; sala de aula com computador e projetor; manequins para treinamento e previsão de materiais e dispositivos para as práticas de habilidades.

As aulas práticas em laboratório com simulação de ambiente real de atendimento ao paciente complementam o alinhamento dos profissionais para construção do conhecimento técnico. ${ }^{(13)}$ Cada grupo de colaboradores admitidos na instituição participa de um ciclo contínuo de novas experiências e acrescenta conhecimentos e envolvimento com a instituição, sentindo-se partícipes de todos seus processos.

\section{Conclusão}

O redesenho do programa de capacitação com a utilização de um referencial teórico como a teoria de Nonaka respaldou o treinamento admissional sistematizado, possibilitando a capacitação, com vistas ao cuidado de qualidade com a criança gravemente enferma, evidenciado pelos bons resultados dos pós-testes de assuntos relacionados com registros de enfermagem, prevenção de infecção por meio da higienização das mãos, cálculo de medicamentos em pediatria e segurança do paciente. Além disso, houve maior envolvimento com o processo admissional planejado e sistematizado explicitado, observando-se a integração e a motivação entre os profissionais submetidos. Faz-se necessário que as instituições formadoras e os serviços de educação permanente detectem as necessidades de treinamento dos profissionais recém-admitidos e elaborem programas educativos sistematizados e teórico-práticos, com metodologias de ensino ativas. Outro aspecto a ser considerado é a humanização, visando ao acolhimento e à ajuda para todos 
os profissionais, principalmente para os que apresentam dificuldades. Desta forma, auxilia-se o colaborador, que praticará o cuidado centrado no paciente.

\section{Referências}

1. Gonçalves JE. A necessidade de reinventar as empresas. Rev Adm Empres. 1998;38(2):6-17.

2. Gonçalves JE. Os novos desafios da empresa do futuro. Rev Adm Empres. 1997;37(3):10-9.

3. Carvalho SR. Os múltiplos sentidos da categoria "empowerment" no projeto de promoção à saúde. Cad Saúde Pública. 2004;20(4):1088-95.

4. Rocha MC, Rossato LM, Misko MD, Bousso RS, Damião EB. Preparando o aluno de pósgraduação para 0 exercício de docência em enfermagem no cuidado da criança e da família na experiência de doença. Rev Soc Bras Enferm Ped. 2010;10(1):31-5.

5. Kurcgant P, Castilho V, Leite MM. Capacitação do profissional de saúde no âmbito da formação e da educação continuada. Rev Esc Enferm USP. 1994;28(3):251-6.

6. Silva LA, Ferraz F, Lino MM, Backes VM, Schmidt SM. Educação permanente em saúde e no trabalho de enfermagem: perspectiva de uma práxis transformadora. Rev Gaúcha Enferm. 2010;31(3):557-61.
7. Brasil. Ministério da Saúde. Secretaria de Gestão do Trabalho e da Educação na Saúde. Departamento de Gestão da Educação em Saúde. Textos Básicos de Saúde - Série Pactos pela Saúde. Brasília (DF): Ministério da Saúde; 2009.

8. Peres C, Silva RF, Barba PC. Desafios e potencialidades do processo de educação permanente em saúde. Trab Educ Saúde. 2016;14(3):783-801.

9. Silva LA, Schmidt SM, Noal HC, Signor E, Gomes IE. Avaliação da educação permanente no processo de trabalho em saúde. Trab Educ Saúde. 2016;14(3):765-81.

10. Nonaka I. A dynamics theory of organizational knowledge creation. Organization Science. 1994;5(1):14-37.

11. Nonaka I, Takeuchi H. Teoria da criação do conhecimento organizacional. In: Nonaka I, Takeuchi H. Criação de conhecimento na empresa: como as empresas japonesas geram a dinâmica da inovação. Rio de Janeiro: Campus; 1997. p. 61-102.

12. Marcondes E, Gonçalves EL. Educação médica. São Paulo: Sarvier; 1998.

13. Ferreira TS. Alinhamento técnico de colaboradores por meio da gestão do conhecimento. São Paulo. Tese [Dissertação] - Faculdade de Economia, Administração e Contabilidade da Universidade de São Paulo; 2019.

14. Silva LB, Gaiotto SA, Igarashi DC. Compreensões da gestão do conhecimento nos níveis tático e operacional de uma organização pública: um estudo de caso. Revista SMG. 2019;7(2)

15. Caldas PT, Firmino TT.A dinâmica de conversão intra e interorganizacional do conhecimento no terceiro setor. XX SEMEAD - Seminários em Administração; 2017; Paraíba: 2017. 